\title{
The redshift and broad-band spectral energy distribution of NRAO 150*
}

\author{
J. A. Acosta-Pulido ${ }^{1,2}$, I. Agudo ${ }^{3,4}$, R. Barrena ${ }^{1,2}$, C. Ramos Almeida ${ }^{1,2,5}$, A. Manchado ${ }^{1,2}$, and P. Rodríguez-Gil ${ }^{1,6}$ \\ 1 Instituto de Astrofísica de Canarias (IAC), C/Vía Láctea, s/n, 38200, La Laguna, Tenerife, Spain \\ e-mail: jap@iac.es \\ 2 Departamento de Astrofísica, Universidad de La Laguna, 38205 La Laguna, Tenerife, Spain \\ 3 Instituto de Astrofísica de Andalucía (CSIC), Apartado 3004, 18080 Granada, Spain \\ ${ }^{4}$ Institute for Astrophysical Research, Boston University, 725 Commonwealth Avenue, Boston, MA 02215, USA \\ 5 Department of Physics \& Astronomy, University of Shefield, UK \\ ${ }^{6}$ Isaac Newton Group of Telescopes, La Palma, Spain
}

Received 23 December 2009 / Accepted 9 March 2010

ABSTRACT

\begin{abstract}
Context. NRAO 150 is one of the brightest radio and mm AGN sources in the northern sky, and is an interesting source to study extreme relativistic jet phenomena. However, its cosmological distance has not yet been calibrated, because of its optical faintness due to strong Galactic extinction.

Aims. We measure the redshift of NRAO 150, to enable us to perform quantitative studies of the source.

Methods. We conducted spectroscopic and photometric observations of the source in both the near-IR and the optical.

Results. All these observations were successful in detecting the source. The near-IR spectroscopic observations exhibit strong H $\alpha$ and $\mathrm{H} \beta$ emission lines from which the cosmological redshift of NRAO $150(z=1.517 \pm 0.002)$ is determined for the first time. We classify the source as a flat-spectrum radio-loud quasar, for which we estimate a high supermassive black-hole mass $\sim 5 \times 10^{9} M_{\odot}$. After extinction correction, the new near-IR and optical data show a high-luminosity continuum-emission excess in the optical (peaking in the rest frame at $\sim 2000 \AA$ ) that we attribute to thermal emission from the accretion disk for which we estimate a high accretion rate of $\sim 30 \%$ the Eddington limit.

Conclusions. Comparison of the source properties and its broad-band spectral-energy distribution with those of Fermi blazars allows us to predict that NRAO 150 is among the most powerful of blazars, and hence a high luminosity - although not yet detected - $\gamma$-ray emitter.
\end{abstract}

Key words. galaxies: active - galaxies: jets - quasars: emission lines - quasars: individual: NRAO 150 - quasars: general

\section{Introduction}

First catalogued by Pauliny-Toth et al. (1966), NRAO 150 is nowadays one of the strongest radio and mm AGN sources in the northern sky (e.g., Teräsranta et al. 2005; Agudo et al. 2010). The source has been monitored at $\mathrm{cm}$ and $\mathrm{mm}$ wavelengths for decades (e.g., Aller et al. 1985; Reuter et al. 1997; Teräsranta et al. 2004, and references therein), and displayed flux densities in the range $[2,16] \mathrm{Jy}$ at $2 \mathrm{~cm}^{1}$ and $[1.5,9.5] \mathrm{Jy}$ at $3 \mathrm{~mm}^{2}$, its absolute maxima having occurred at the beginning of year 2009.

At radio wavelengths, NRAO 150 displays on VLBI scales, a compact core plus a one-sided jet extending up to $r \gtrsim 80$ mas with a jet structural position angle (PA) of $\sim 30^{\circ}$ (e.g., Fey \& Charlot 2000). The first set of ultra-high-resolution mm-VLBI images of NRAO 150 (Agudo et al. 2007) allowed them to detect a large misalignment $\left(>100^{\circ}\right)$ between the $\mathrm{cm}$-wave and the $\mathrm{mm}$-wave jet, which in addition to the one sidedness of the jet, is a clear sign of jet orientation close to the line of sight. There

* Based on observations made with the William Herschel Telescope operated on the island of La Palma by the Isaac Newton Group in the Spanish Observatorio del Roque de los Muchachos of the Instituto de Astrofísica de Canarias.

${ }^{1}$ http://www.astro.1sa. umich. edu/obs/radiotel

${ }^{2} \mathrm{H}$. Ungerechts, private communication. has also been evidence of rapid "jet wobbling" at $\sim 11^{\circ} / \mathrm{yr}$ in the plane of the sky, which is the most rapid for an AGN found so far. The observations by Agudo et al. (2007) together with the cosmological redshift measurement presented in this paper and non-contemporaneous X-ray data have allowed them to report the first quantitative estimates of the basic physical properties of the inner jet in NRAO 150, i.e., Doppler factor $\delta \approx 6$, the bulk Lotentz factor $\gamma \approx 4$, the angle subtended between the jet and the line of sight $\phi \approx 8^{\circ}$, and the magnetic field intensity of the flow $B \approx 0.7 \mathrm{G}$. This high $B$ estimate seems to be compatible with the highly non-balistic superluminal motion of the inner jet in the source detected by the mm-VLBI images $\left(\beta_{\text {app }} \approx 3\right.$ times the speed of light), which have also demonstrated that NRAO 150 is a prime target for studying the origin of the jet wobbling phenomenon (Agudo 2009).

In the optical, NRAO 150 was first detected in December 1981 by Landau et al. (1983). However, no optical classification or distance determination has been reported so far, perhaps because of the difficulties in observing the source at visible wavelengths, where NRAO 150 is strongly affected by Galactic extinction (Galactic latitude $b \approx-1.6^{\circ}$ ). This problem is partially overcome in the near-IR range, where spectroscopic observations from strong-lined objects can be performed from Earth. Independent of the Galactic absorption along the line of sight 
to the source, the near-IR range is an adequate spectral range for detecting the strong $\mathrm{H} \alpha$ line from AGN at cosmological redshifts between 1.2 and 3.6 (e.g., Babbedge et al. 2004).

Here, we present the results of our spectroscopic near-IR observations, which were successful in detecting, for the first time, emission lines from NRAO 150. In Sect. 2, these observations and their data reduction procedures are outlined and applied to a set of optical and near-IR photometric observations performed in the 2005-2007 time span. The cosmological redshift determination, the classification, and the first estimate of the mass of the supermassive compact object in NRAO 150 and both its accretion rate and broad-band spectral energy distribution, are presented and discussed in Sects. 3 and 4, whereas a summary of our main results and conclusions are provided in Sect. 5.

\section{Observations and data reduction}

\subsection{Near infrared spectroscopy}

We obtained near-IR spectroscopy of NRAO 150 using the Long-slit Intermediate Resolution Infrared Spectrograph (LIRIS, built at the Instituto de Astrofísica de Canarias Manchado et al. 2004; Acosta-Pulido et al. 2003) on the $4.2 \mathrm{~m}$ William Herschel Telescope (WHT) in two epochs (2005 March and 2007 January).

The spectra were obtained using grisms LR_ZJ (bands $Z+J$ ) and LR_HK (bands $H+K$ ). For the observations in 2005, the slit width was selected to be 0.75 , providing resolutions of 700 and 600 in the $Z+J$ and $H+K$ bands, respectively. For those in 2007, the slit width was $1^{\prime \prime}$ to match the seeing, providing a resolution of 500 in the $Z+J$ bands. The slit orientation $\left(\mathrm{PA}=118.8^{\circ}\right)$ was chosen to include in the slit aperture both NRAO 150 and the object 2MASS J03592889+5057547. The observations were performed following an ABBA telescope nodding pattern, each $\mathrm{AB}$ cycle being repeated 3 times. The exposure time for a single frame was $600 \mathrm{~s}$, giving a total of $3600 \mathrm{~s}$ for both spectral ranges. The data were reduced following standard recipes for near-IR spectroscopy, using the dedicated software lirisdr, developed within the IRAF ${ }^{3}$ enviroment by the LIRIS team. The basic reduction steps comprise sky subtraction, flat-fielding, wavelength calibration, and finally, the combination of individual spectra by the common shift-and-add technique. For a more detailed description of the reduction process, we refer to Ramos Almeida et al. (2006, 2009). The flux calibration and telluric absorption correction are usually determined from observations of nearby stars of spectral types A0V or G2V, which are obtained inmediately before or after the science observations. In the first epoch, the selected correction star was found to be a multiple stellar system whose 2MASS near-IR colors indicate a spectral type that differs from A0. We determined a spectral response function using the spectrum of the star 2MASS J03592889+5057547, which was observed simultaneously with NRAO 150. The stellar spectral shape was corrected by a black body of temperature corresponding to an M5 spectral type. A posteriori, we derived the telluric correction from the normalized spectrum of star 2MASS J03592889+5057547. During the second epoch, an A0V star was successfully observed. A modified version of the Xtellcor routine was used (Vacca et al. 2003) to obtain the calibrated flux and telluric-corrected NRAO 150 spectrum. Relative light losses due to atmospheric differential refraction are very small, always

\footnotetext{
3 IRAF (Image Reduction and Analysis Facility) is distributed by the National Optical Astronomy Observatories, which are operated by AURA, Inc., under cooperative agreement with the National Science Foundation.
}

below a few percent, despite the slit not being oriented at the parallactic angle. The flux calibrated spectra are presented in Fig. 1.

\subsection{Near infrared photometry}

We obtained images in the $J$ and $K_{\mathrm{s}}$ filters using LIRIS at the same epochs as the near-IR spectroscopy. In both cases, the images were obtained following a 5 point dithering pattern. Individual frames of 20 and $10 \mathrm{~s}$ were taken in the $J$ and $K_{\mathrm{s}}$ filters, respectively, providing the total exposure times reported in Table 2. The images were reduced using the task ldedither included in the dedicated software package lirisdr, developed in IRAF by the LIRIS team. The main data reduction steps are sky subtraction, flat-field correction, and finally a combination of the images after proper alignment.

The photometric calibration was determined by comparing with the 2-MASS catalogue (Cutri et al. 2003). The zero points of our $J$ and $K_{\mathrm{s}}$ photometry were determined using about 20 stars with known 2-MASS magnitudes (brighter than $J \sim 15.5$ ) included in the LIRIS field. The typical dispersion between instrumental and 2-MASS magnitudes is $\sim 0.08$.

\subsection{Optical photometry}

In addition to the near-IR observations, we also obtained images in the optical using several telescopes at different epochs. The first observations were performed using the CCD camera mounted on the IAC- 80 at the Teide Observatory during the nights of 2005 Nov. 2 and 11. We used filters $V, R$, and $I$ (see Table 1). Our target was detected in all bands (see Fig. 2 for examples of $I$ and $V$ images). Given the faintness of the source, a larger diameter telescope, the $2.5 \mathrm{~m}$ Liverpool robotic telescope (Steele et al. 2004) was used to perform the remaining observations. Filters Johnson $V$ and Sloan $r^{\prime}$ and $i^{\prime}$ were used, although most data were obtained only in $r^{\prime}$ (see Table 1).

All data were reduced in IRAF following the standard procedures, i.e., bias subtraction, flat-fielding, and image combination. The photometric calibration was based on images of Landolt fields obtained in photometric conditions. The photometry was obtained using the SExtractor program (Bertin \& Arnouts 1996) and the AUTOMAG parameter. The resulting photometric measurements are listed in Table 2. We estimated that the detection of faint objects in our images is complete to $V=23.1$ (24.0) mag, $r^{\prime}=21.7$ (23.0) mag, and $i^{\prime}=20.4$ (21.5) mag for $S / N=5$ (3) within the observed fields.

Based on our photometric calibration, we propose seven reference stars for $r^{\prime}$ and $i^{\prime}$ filters to be used in future monitoring. These stars are marked in Fig. 2 and their corresponding magnitudes are listed in Table 3.

\section{Results}

\subsection{Near IR spectrum}

A very prominent feature is observed in the spectrum of NRAO 150 in the $H$ band at $1.65 \mu \mathrm{m}$ (see Fig. 1). A less intense, but notable, feature is also seen in the $J$ band at $\sim 1.23 \mu \mathrm{m}$. These are identified with the $\mathrm{H} \alpha$ and $\mathrm{H} \beta$ lines, respectively, redshifted by $z=1.517 \pm 0.002$ providing the first redshift determination of NRAO 150. The corresponding luminosity distance of the source is $d_{\mathrm{L}}=11.2 \times 10^{4} \mathrm{Mpc}$, for a $H_{0}=71 \mathrm{~km} \mathrm{~s}^{-1} \mathrm{Mpc}^{-1}, \Omega_{\mathrm{m}}=0.27$, and $\Omega_{\Lambda}=0.73$ cosmology 4 .

\footnotetext{
${ }^{4}$ The cosmology calculator available in the Web (http://wWw. astro.ucla.edu/ wright/CosmoCalc.html) was used.
} 
J. A. Acosta-Pulido et al.: The redshift and SED of NRAO 150
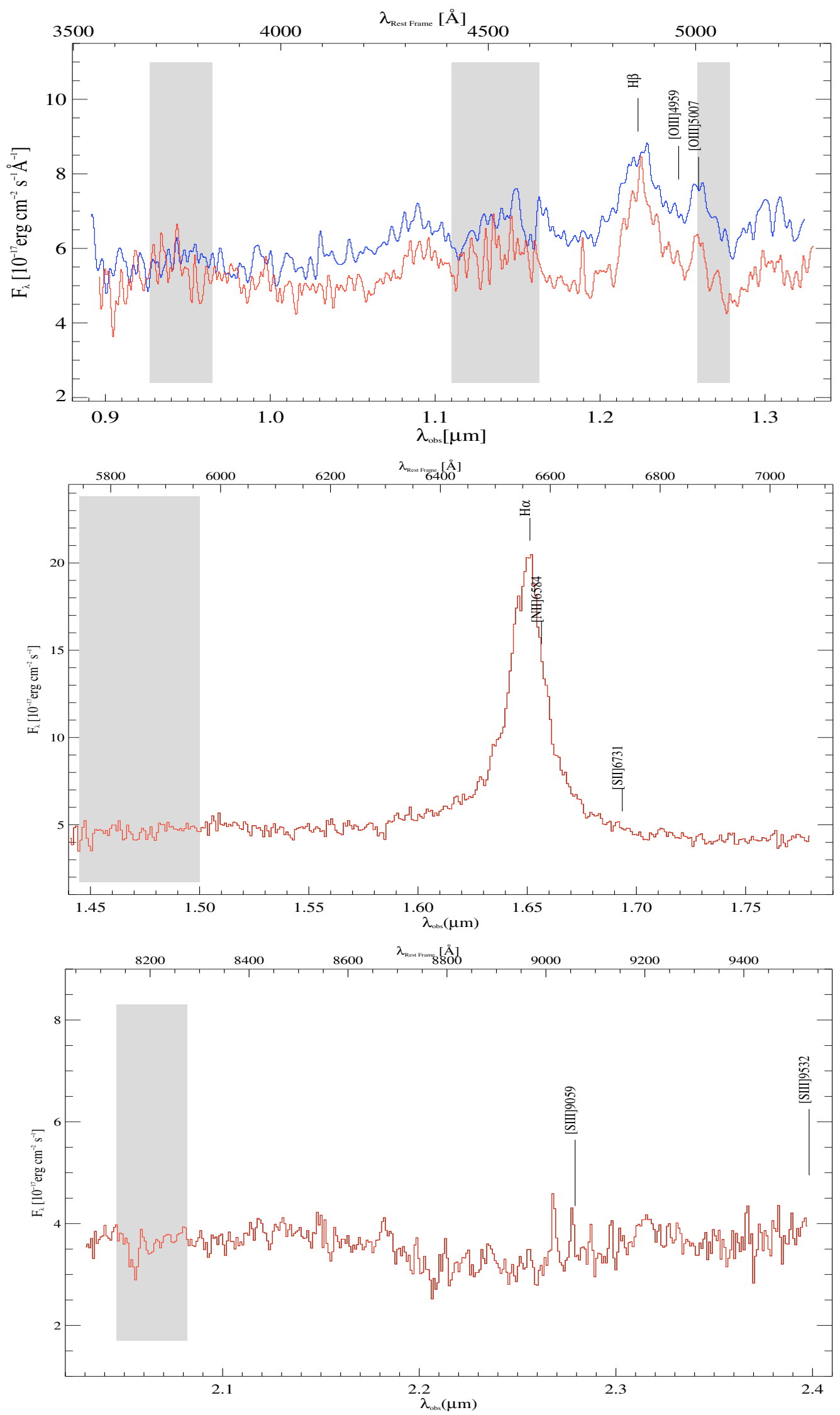

Fig. 1. Final reduced spectrum of NRAO 150. The position of the most prominent features are marked. Deep atmospheric absorption bands are indicated by vertical bands. Top panel: spectra corresponding to the bands $Z+J$ observed at two epochs. The thicker red color line corresponds to observations performed in March 2005, and the thinner blue color line corresponds to those of January 2007. Middle and bottom panels: spectra corresponding to the bands $H$ and $K$, respectively. 
Table 1. Observing log.

\begin{tabular}{|c|c|c|c|c|}
\hline \multicolumn{5}{|c|}{ SPECTROSCOPY } \\
\hline Date & $\begin{array}{c}\text { Spec range } \\
\mu \mathrm{m}\end{array}$ & $\begin{array}{c}\text { Dispersion } \\
\AA / \text { pix }\end{array}$ & $\begin{array}{c}T_{\exp } \\
\mathrm{S}\end{array}$ & $\begin{array}{l}\text { Seeing } \\
\operatorname{arcsec}\end{array}$ \\
\hline 2005 March 23 & $0.89-1.33$ & 6.1 & 3600 & 0.7 \\
\hline 2005 March 22 & $1.45-2.40$ & 9.6 & 3600 & 0.7 \\
\hline 2007 Jan. 2 & $0.89-1.33$ & 6.1 & 3600 & 1 1.2 \\
\hline 2007 Jan. 4 & $0.89-1.33$ & 6.1 & 2400 & 1 1.2 \\
\hline \multicolumn{5}{|c|}{ PHOTOMETRY } \\
\hline Date & Instrum/Teles & c $\quad$ Filter & $T_{\exp }(\mathrm{s})$ & Seeing \\
\hline \multirow[t]{2}{*}{2005 March 22} & LIRIS/WHT & $J$ & 400 & 1". 4 \\
\hline & & $K_{\mathrm{s}}$ & 400 & 1." 4 \\
\hline \multirow[t]{3}{*}{2005 Nov. 2} & IAC-80 & $V$ & 9000 & 1." 5 \\
\hline & & $R$ & 6600 & $1 " .3$ \\
\hline & & $I$ & 6000 & 1 ". 3 \\
\hline \multirow[t]{2}{*}{2005 Nov. 11} & IAC- 80 & $R$ & 3600 & 1.". 4 \\
\hline & & $I$ & 4800 & 1." 4 \\
\hline \multirow[t]{3}{*}{2006 Feb. 03} & Liv. Tel. & V & 600 & 1.. 4 \\
\hline & & $r^{\prime}$ & 300 & 1."2 \\
\hline & & $i^{\prime}$ & 200 & 1 ". 2 \\
\hline \multirow[t]{2}{*}{2006 Feb. 17} & Liv. Tel. & $r^{\prime}$ & 1800 & 1.. 1 \\
\hline & & $i^{\prime}$ & 900 & 1.0 \\
\hline \multirow[t]{2}{*}{2006 Feb. 20} & Liv. Tel. & $r^{\prime}$ & 1800 & $1 " .3$ \\
\hline & & $i^{\prime}$ & 900 & 1.22 \\
\hline 2006 Feb. 22 & Liv. Tel. & $r^{\prime}$ & 1400 & 0.9 \\
\hline 2006 Aug. 22 & Liv. Tel. & $r^{\prime}$ & 1400 & 0.9 \\
\hline 2006 Sep. 18 & Liv. Tel. & $r^{\prime}$ & 1400 & 0.9 \\
\hline 2006 Sep. 30 & Liv. Tel. & $r^{\prime}$ & 1400 & 0.9 \\
\hline 2006 Oct. 21 & Liv. Tel. & $r^{\prime}$ & 1400 & 0.9 \\
\hline 2006 Nov. 13 & Liv. Tel. & $r^{\prime}$ & 1400 & 0.9 \\
\hline 2006 Nov. 22 & Liv. Tel. & $r^{\prime}$ & 1400 & 0.9 \\
\hline \multirow[t]{2}{*}{2006 Dec. 30} & LIRIS/WHT & $J$, & 300 & 1.. 1 \\
\hline & & $K_{\mathrm{s}}$ & 250 & 1." 1 \\
\hline 2007 Jan. 8 & Liv. Tel. & $r^{\prime}$ & 1400 & $0 ! 9$ \\
\hline 2007 Jan. 14 & Liv. Tel. & $r^{\prime}$ & 1400 & 0.9 \\
\hline 2007 Jan. 21 & Liv. Tel. & $r^{\prime}$ & 1400 & 0.9 \\
\hline
\end{tabular}

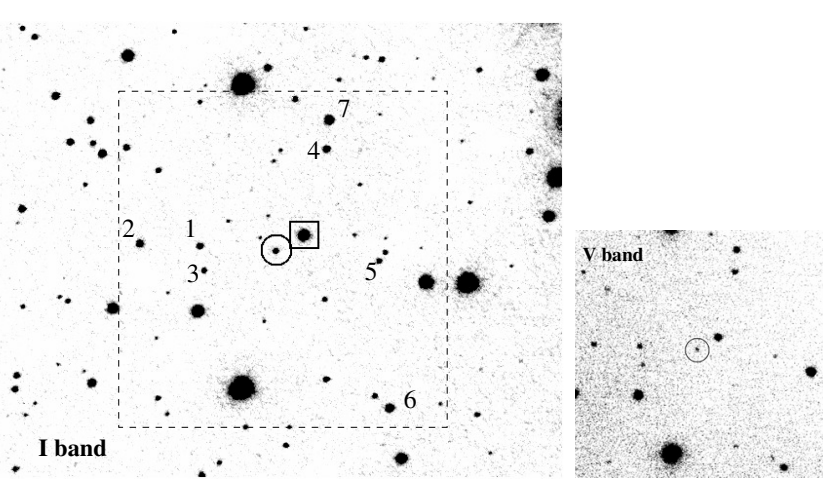

Fig. 2. Identification charts of NRAO 150 in $I$ and $V$ filters. The circle and square symbols mark NRAO 150 and the star 2MASS J03592889+505754, used as reference for spectroscopy. Dashed square represent the plotted area in $V$ band. North and east are toward the top and left of the frame, respectively. Numbers note the calibration stars proposed for this field.

We measured the center, width, and flux of the narrow and broad components of $\mathrm{H} \alpha$ and $\mathrm{H} \beta$ by fitting two Gaussian components plus a slope as the continuum (see Table 4). We started by fitting $\mathrm{H} \alpha$ since this part of the spectrum has a higher signalto-noise ratio and is not contaminated by other feature, such as FeII emission. The $\mathrm{H} \alpha$ profile is accurately fitted by a narrow component of $F W H M=170 \AA\left(1458 \mathrm{~km} \mathrm{~s}^{-1}\right.$ rest-frame $)$ and a
Table 2. NRAO 150 - Optical near-IR photometry.

\begin{tabular}{llllcc}
\hline \hline Date & JD & \multicolumn{2}{c}{$J$} & \multicolumn{2}{c}{$K_{\mathrm{s}}$} \\
\hline 1999 Oct. 13 & 51465 & $16.49 \pm 0.12^{*}$ & $14.37 \pm 0.08^{*}$ \\
2005 Mar. 23 & 53453 & $16.78 \pm 0.06$ & $14.81 \pm 0.05$ \\
2006 Dic. 30 & 54100 & $16.52 \pm 0.04$ & $14.54 \pm 0.05$ \\
\hline \multicolumn{5}{c}{ Optical } \\
\hline Date & JD & \multicolumn{5}{c}{$r^{\prime}$} & $i^{\prime}$ \\
\hline 2005 Nov. 2 & 53677.45 & $22.9 \pm 0.9$ & $20.67 \pm 0.08$ & $19.43 \pm 0.10$ \\
2005 Nov. 11 & 53683.4 & $\ldots$ & $20.81 \pm 0.07$ & $19.72 \pm 0.04$ \\
2006 Feb. 3 & 53770.35 & $\ldots$ & $20.84 \pm 0.04$ & $19.19 \pm 0.02$ \\
2006 Feb. 17 & 53784.35 & $\ldots$ & $20.58 \pm 0.03$ & $19.32 \pm 0.05$ \\
2006 Feb. 20 & 53787.48 & $\ldots$ & $20.60 \pm 0.06$ & $19.68 \pm 0.05$ \\
2006 Feb. 22 & 53789.38 & $\ldots$ & $20.70 \pm 0.05$ & $19.57 \pm 0.06$ \\
2006 Aug. 22 & 53970.65 & $\ldots$ & $20.72 \pm 0.10$ & $\ldots$ \\
2006 Sep. 18 & 53997.51 & $\ldots$ & $20.88 \pm 0.02$ & $\ldots$ \\
2006 Sep. 30 & 54009.56 & $\ldots$ & $20.60 \pm 0.05$ & $\ldots$ \\
2006 Oct. 21 & 54030.46 & $\ldots$ & $20.62 \pm 0.01$ & $\ldots$ \\
2006 Nov. 13 & 54053.39 & $\ldots$ & $20.68 \pm 0.02$ & $\ldots$ \\
2006 Nov. 22 & 54062.44 & $\ldots$ & $20.78 \pm 0.02$ & $\ldots$ \\
2007 Jan. 8 & 54109.38 & $\ldots$ & $20.85 \pm 0.01$ & $\ldots$ \\
2007 Jan. 14 & 54115.35 & $\ldots$ & $20.67 \pm 0.04$ & $\ldots$ \\
2007 Jan. 21 & 54122.40 & $\ldots$ & $20.81 \pm 0.09$ & $\ldots$ \\
\hline
\end{tabular}

Notes. ${ }^{(*)}$ Values corresponding to 2-MASS PSC.

broad component of $F W H M=666 \AA\left(5745 \mathrm{~km} \mathrm{~s}^{-1}\right.$ rest-frame $)$. The broad component is blueshifted with respect to the narrow component by $62 \AA\left(532 \mathrm{~km} \mathrm{~s}^{-1}\right)$. In the case of $\mathrm{H} \beta$ the $\mathrm{S} / \mathrm{N}$ ratio is much lower and we were unable to allow the line widths to vary in the fitting. Instead they were constrained by the best fit solution for $\mathrm{H} \alpha$.

Other broad features of lower $\mathrm{S} / \mathrm{N}$ are observed around $4300 \AA$ (rest frame), corresponding to the Balmer limit, and at 4450-4700, 4924, 5018 and 5150-5350 A, associated with blends of FeII emission (see Fig. 1). To remove and confirm the importance of FeII emission, we subtracted the empirical FeII emission template of Boroson \& Green (1992), scaled to the intensity of the observed features (see Fig. 3). This template was generated from a spectrum of PG $0050+124$ (IZw 1), which is distinctive because of the strength of its FeII emission and the narrowness of its H recombination lines (Oke \& Lauer 1979). The template (kindly provided by Dr. P. Marziani) was prepared by removing the lines not associated with Fe II transitions. From Fig. 3, it can be seen that after the FeII template subtraction the residual spectra exhibits uniquely the broad $\mathrm{H} \beta$ feature, and a shallow bump at $4300 \AA$ corresponding to the Balmer limit. Given the radio loud AGN properties of NRAO 150, it is expected to observe intense narrow forbidden emission lines, such as [OIII] $\lambda 5007 \AA$, [NII] $\lambda 6548 \AA, \lambda 6584 \AA$, and [SII] $\lambda \lambda 6716$, $6731 \AA$. However, none of these features are detected at a significant level over the continuum. The implications of weak or absent typical NLR features are discussed in Sect. 4.2.

\subsection{Optical and near IR variability}

Our optical and near-IR photometric measurements span a range of almost 2 years (see Table 2), which is typically a sufficiently long time range to detect intrinsic variability in radio-loud AGN (e.g., Teräsranta et al. 2004, 2005). NRAO 150 exhibits a nearly monotonic radio flux increase by a factor $\sim 4$ from 1991 to 2005 (Teräsranta et al. 2004, 2005). However, we cannot make any firm claim about optical variability based on measurements in 
Table 3. Photometry of reference stars near NRAO 150.

\begin{tabular}{lccccc}
\hline \hline ID & 2MASS-ID & $\begin{array}{c}\text { RA } \\
(03: 59: \mathrm{ss})\end{array}$ & $\begin{array}{c}\text { Dec }(\mathrm{J} 2000) \\
\left(+50:^{\prime}:^{\prime \prime}\right)\end{array}$ & $r^{\prime}$ & $i^{\prime}$ \\
\hline 1 & $\mathrm{~J} 03593205+5057513$ & 32.05 & $57: 51.4$ & $20.12 \pm 0.04$ & $19.10 \pm 0.07$ \\
2 & $\mathrm{~J} 03593386+5057522$ & 33.87 & $57: 52.2$ & $20.12 \pm 0.02$ & $18.48 \pm 0.01$ \\
3 & $\ldots$ & 31.86 & $57: 45.0$ & $20.86 \pm 0.10$ & $19.79 \pm 0.14$ \\
4 & $\mathrm{~J} 03592824+5058196$ & 28.24 & $58: 19.6$ & $19.62 \pm 0.01$ & $18.66 \pm 0.07$ \\
5 & $\ldots$ & 56.58 & $57: 47.3$ & $21.00 \pm 0.07$ & $19.75 \pm 0.14$ \\
6 & $\mathrm{~J} 03592625+5057054$ & 26.26 & $57: 05.1$ & $18.98 \pm 0.01$ & $18.04 \pm 0.09$ \\
7 & $\mathrm{~J} 03592814+5058277$ & 28.18 & $58: 27.7$ & $18.76 \pm 0.01$ & $17.70 \pm 0.04$ \\
\hline
\end{tabular}

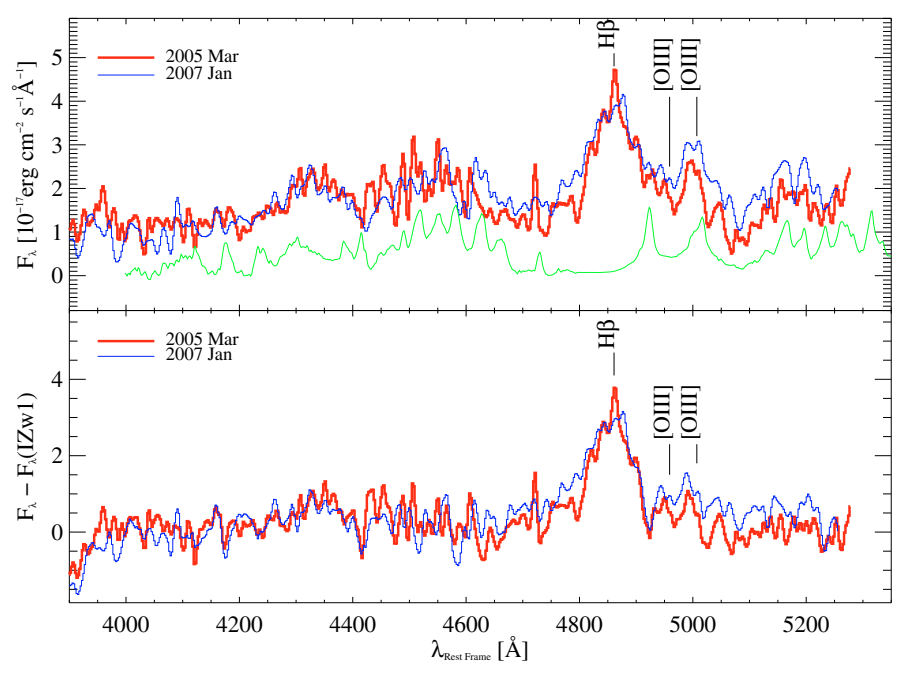

Fig. 3. Top panel: the two NRAO 150 spectra (histogram style line) taken at different epochs are represented together with a scaled version of the IZw1 template (green continuous thin line). Bottom panel: the spectra after subtraction of the template. It can be seen that the residual spectra consist basically on a broad $\mathrm{H} \beta$ feature, a shallow bump at $4300 \AA$ A corresponding to the Balmer jump, and very weak [OIII] lines.

the $r^{\prime}$ band, the band with by far the longer and more frequently sample coverage in terms of time. The maximum magnitude variations observed are $\sim 0.2 \mathrm{mag}$, which are not larger than 3 times the typical measurement uncertainty. We note that part of the data were taken using different combinations of filters, cameras, and telescopes, which does not enhance their homogeneity. The faintness of the source in the optical range also prevents us from obtaining more accurate photometric measurements.

As in the optical range, our near-IR photometric measurements (performed in 2005 March and 2006 December) do not exhibit significant variability of amplitude above $\sim 0.3$ mag $(\sim 0.25$ or $30 \%)$. A similar result is obtained when our near-IR measurements are compared with those of the 2-MASS survey in 1999 (see Table 2). In this case, maximum variability amplitudes of $\Delta J=0.3 \mathrm{mag}$ and $\Delta K_{\mathrm{S}}=0.4 \mathrm{mag}$ are found, which contrasts with the factor of 2 flux density increase reported by Teräsranta et al. (2005) at radio wavelengths for the same time range. This suggests that the process responsible for the radio emission is not connected to that responsible for the optical and near IR emission.

\section{Discussion}

\subsection{Black hole mass and its accretion efficiency}

We use the empirical relationships provided by Vestergaard \& Peterson (2006) to estimate the central black hole mass in
NRAO 150. The black hole (BH) mass can be estimated from the luminosity and width of the broad component of the $\mathrm{H} \beta$ line using expresion 6 in Vestergaard \& Peterson (2006). Given the low $\mathrm{S} / \mathrm{N}$ ratio of the $\mathrm{H} \beta$ line detected in our spectral measurements, the use of the line luminosity is preferable to the value of the continuum at $5100 \AA$ (used in Eq. (5) of Vestergaard \& Peterson 2006). The measured broad component luminosity is $5.6 \pm 1.2 \times 10^{43} \mathrm{erg} \mathrm{s}^{-1}$, which increases to $2.3 \pm 0.5 \times 10^{44} \mathrm{erg} \mathrm{s}^{-1}$ after applying the Galactic extinction correction derived in this work (see Appendix A). Combining these values with those for the line width (see Table 4), the resulting black hole mass is $1.95 \times 10^{9} M_{\odot}$, which converts to $4.68 \times 10^{9} M_{\odot}$ after extinction correction. Using the estimate of the black hole mass, we can also determine the corresponding Eddington luminosity $\left[L_{\mathrm{Edd}}=3.3 \times 10^{4}\left(M_{\mathrm{BH}} / M_{\odot}\right) L_{\odot}\right]$, which results in a value of $1.54 \times 10^{14} L_{\odot}$. To compute the accretion rate, this value must be compared with the bolometric luminosity of the accretion disk, which results in $L_{\mathrm{disk}} \simeq 7.8 \times 10^{12} L_{\odot}$, and $L_{\mathrm{disk}} \simeq 46.9 \times 10^{12} L_{\odot}$ after extinction correction. The disk luminosity was computed by integrating the measured fluxes within the optical-UV range in the rest frame, this value is multiplied by $4 \pi D^{2}$ to obtain the luminosity. Here it is assumed that the radio-mm and the X-ray emission are related to the relativistic jet. This is justified given the variability in the optical-UV spectral ranges, which is uncoupled with that in the radio-mm as reported in Sect. 3.2. Thus the Eddington luminosity ratio is $L_{\text {disk }} / L_{\text {Edd }}=0.30$.

These values can be compared to those previously reported in the literature for similar objects. The black hole mass of NRAO 150 is above the highest masses found in the literature for low redshift AGNs (Vestergaard \& Peterson 2006), although the estimated value for M 87 , is $3.4 \times 10^{9} M_{\odot}$ (Graham 2007), comparable to that of NRAO 150. In contrast, the black hole mass of NRAO 150 is well within the range of typical masses of luminous high redshift AGNs (Shemmer et al. 2004). Several works (e.g., McLure \& Dunlop 2004; Shen et al. 2008) have tried to measure the dependence of the mean $\mathrm{BH}$ mass on redshift. They reach a common result: the mean $\mathrm{BH}$ mass increases with redshift, although this dependence is dominated by the Malmquist bias (Vestergaard et al. 2008). Labita et al. (2009) claimed that the maximum BH mass evolves with $z$ as $\log \left(M_{\mathrm{BH}(\max )} / M_{\odot}\right) \sim 0.3 z+9$, whereas the maximum Eddington ratio $(0.45)$ is essentially constant with $z$. However, Labita et al. (2009) suggest that these results are unaffected by the Malmquist bias. Our estimate for the BH mass in NRAO 150 is slightly above the maximum value, and the Eddington ratio is also close to the maximum value, which indicates that NRAO 150 contains a very massive and efficiently accreting $\mathrm{BH}$. 
Table 4. Flux units are $10^{-15} \mathrm{erg} \mathrm{s}^{-1} \mathrm{~cm}^{-2}$. Luminosity units are $10^{43} \mathrm{erg} \mathrm{s}^{-1}$.

\begin{tabular}{lccccccc}
\hline \hline & Center & \multicolumn{2}{c}{$F W H M$} & \multicolumn{2}{c}{ Flux } & \multicolumn{2}{c}{ Lum. } \\
Line & $(\AA)$ & $(\AA)$ & $\mathrm{km} \mathrm{s}^{-1}$ & Obs. & Ext. Cor. & Obs. & Ext. Cor. \\
\hline $\mathrm{H} \alpha(\mathrm{n})$ - 2005 March & $16501.1 \pm 0.7$ & $72 \pm 1.7$ & 1458 & $22.0 \pm 0.5$ & $51.7 \pm 1.2$ & $32.9 \pm 0.7$ & $77.1 \pm 1.8$ \\
$\mathrm{H} \alpha(\mathrm{b})$ - 2005 March & $16439 \pm 7$ & $283 \pm 16$ & 5745 & $21.7 \pm 0.7$ & $51.0 \pm 1.6$ & $32.4 \pm 1.0$ & $76.1 \pm 2.4$ \\
$\mathrm{H} \beta(\mathrm{n})$ - 2005 March & $12255 \pm 5$ & $54 \pm 1.2$ & 1458 & $2.97 \pm 0.3$ & $12.0 \pm 1.2$ & $4.4 \pm 0.4$ & $17.9 \pm 0.6$ \\
$\mathrm{H} \beta(\mathrm{b})$ - 2005 March & $12209 \pm 5$ & $210 \pm 12$ & 5745 & $3.74 \pm 0.8$ & $15.1 \pm 3.2$ & $5.6 \pm 1.2$ & $23 \pm 5$ \\
$\mathrm{H} \beta(\mathrm{n})$ - 2007 Jan. & $12254 \pm 9$ & $54 \pm 1.2$ & 1458 & $2.14 \pm 0.4$ & $8.6 \pm 1.6$ & $3.2 \pm 0.6$ & $13 \pm 2$ \\
$\mathrm{H} \beta(\mathrm{b})$ - 2007 Jan. & $12208 \pm 9$ & $210 \pm 12$ & 5745 & $3.45 \pm 0.9$ & $13.9 \pm 3.6$ & $5.1 \pm 1.3$ & $21 \pm 5$ \\
\hline
\end{tabular}

\subsection{Fell emission and weakness of the NLR emission features}

As reported above, the spectrum of NRAO 150 presents both prominent $\mathrm{H}$ recombination lines and intense FeII emission, in contrast to the very weak or absent lines characteristic of the NLR, such as [OIII] (see Fig. 3). Netzer et al. (2004) found that about one third of very high luminosity AGNs do not exhibit strong [OIII] lines. In addition, an anticorrelation between $\mathrm{EW}(\mathrm{FeII}) / \mathrm{EW}(\mathrm{H} \beta)$ and $\mathrm{EW}([\mathrm{OIII}])$ (Boroson \& Green 1992; Yuan \& Wills 2003; Netzer et al. 2004) is reported. In our NRAO 150 spectra, the estimated EWs of $\mathrm{H} \beta$, FeII, and [OIII] are around 55, 60, and 6, although the uncertainties are large given the difficulty in deblending the spectral features. These values are consistent with the afore mentioned results. Using these values, we checked that NRAO 150 is placed in Fig. 9 of Netzer et al. (2004) on the locus of high luminosity and highredshift QSO sample. The location of NRAO 150 in Fig. 3 of Netzer et al. (2004) is in-between that of PG QSOs (Bennert et al. 2002) and $z>2$ QSOs in terms of its $\mathrm{H} \beta$ luminosity. Netzer et al. (2004) suggest that for highly luminous QSOs the NLR becomes extremely large (even larger than the size of the biggest galaxies) as expected from the "natural" $R_{\mathrm{NLR}} \propto L_{\text {ion }}^{1 / 2}$ dependence, which makes the NLR to disappear and become dynamically unbound. The equivalent size of the NLR in NRAO 150, as predicted from the $\mathrm{H} \beta$ luminosity $\left(\sim 4 \times 10^{44} \mathrm{erg} \mathrm{s}^{-1}\right)$, is about $20 \mathrm{kpc}$ (Fig. 3 in Netzer et al. 2004), which is well above the sizes commonly reported for typical NLR, hence explaining the weakness of the NLR emission features in our spectra.

\subsection{The spectral energy distribution}

Figure 4 shows the spectral energy distribution (SED) of NRAO 150. In addition to the photometric data presented here, we searched for data covering the widest possible wavelength range, from radio to X-rays. At radio bands, measurements of the lowest frequency, obtained at $4,8.4,22$, and $43 \mathrm{GHz}$ with the VLA on March 2005, were taken from the National Radio Astronomy Observatory ${ }^{5}$ data archive. The subsequent three measurements were observed at 86,142 , and $229 \mathrm{GHz}$ as part of the general IRAM 30-m Telescope AGN monitoring program (Reuter et al. 1997, and references therein) on March 2005 (August 2005 for the higher frequency one). The optical-UV rest-frame observations corresponds to the near-IR and the optical observations presented here, and were acquired on March 2005 and November 2005, respectively. In this spectral region, empty triangles indicate the extinction-uncorrected optical and near-IR measurements. Empty circles correspond to these measurements corrected for the Galactic extinction estimated from

\footnotetext{
5 The National Radio Astronomy Observatory is a facility of the National Science Foundation operated under cooperative agreement by Associated Universities, Inc.
}

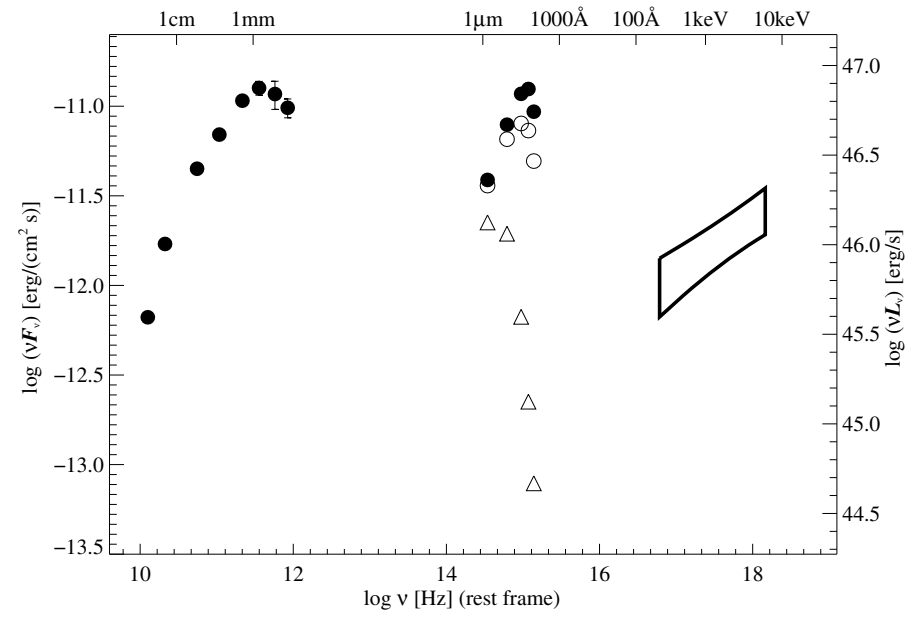

Fig. 4. Spectral energy distribution (SED) of NRAO 150 from the radio to the X-ray spectral range after correction for Galactic extinction. Filled circles represent Galactic extinction estimated in this work (see Appendix A), and empty circles represent the extinction estimated from IRAS far-IR emission maps (Schlegel et al. 1998). Empty triangles indicate the extinction-uncorrected optical and near-IR measurements, and we note the dramatic change of the SED after extinction correction is applied. The measurements presented here were obtained almost contemporaneously during year 2005 (see text), except for the ROSAT X-ray data (bow tie), which were acquired between August 1990 and February 1991.

IRAS far-IR emission maps (Schlegel et al. 1998), whereas filled circles indicate the same measurements corrected for the more accurate Galactic extinction estimated here (see Appendix A). The X-ray data, acquired by ROSAT from August 1990 and February 1991, were corrected by Galactic extinction as reported in Agudo et al. (2007). For the plot in Fig. 4, a photon index $\Gamma=1.7 \pm 0.1$ was assumed based on the synchrotron spectrum measured from the IRAM $30 \mathrm{~m}$ observations presented here at 86 and $142 \mathrm{GHz}$ (spectral index $\alpha=0.7 \pm 0.1$ ).

The SED of NRAO 150 exhibits two prominent bumps: one peaking around $1 \mathrm{~mm}$ and another at $2000 \AA$. The low frequency SED bump resembles those typical of high power flatspectrum radio-loud sources (e.g., Ghisellini et al. 2009a,b). This first bump is attributed to strong Doppler-boosted synchrotron relativistic-jet-emission. The X-ray domain of the SED is also typical of inverse Compton emission from the jet, whereas the high luminosity optical-UV bump $\left(L \approx 10^{47} \mathrm{erg} / \mathrm{s}\right.$ at $v \approx$ $10^{14.5} \mathrm{~Hz}$ ) is not so commonly reported for this kind of source. A prominent peak at optical-UV (rest frame) wavelengths is typical of Seyfert galaxies and is understood to be produced by thermal emission from the AGN accretion disk. However, there is an increasing number of high-power radio-loud blazars for 
which this emission feature is being reported (e.g., Raiteri et al. 2007; Abdo et al. 2009; D’Ammando et al. 2009; Ghisellini \& Tavecchio 2009; Ghisellini et al. 2009a,b). Nearly half of the radio loud blazars in the sample considered by Ghisellini et al. (2009a) were reported to exhibit a similar optical-UV large excess. There is a consensus toward considering this emission to come from thermal emission from the accretion disk (e.g., Raiteri et al. 2007; Abdo et al. 2009; D'Ammando et al. 2009; Ghisellini \& Tavecchio 2009; Ghisellini et al. 2009a,b), which is not overwhelmed by the synchrotron bump from the jet when this bump peaks at $v \ll 10^{14.5} \mathrm{~Hz}$. We also agree that this is a likely explanation for NRAO 150 because of the reasons outlined by Ghisellini et al. (2009b). This also explains the abovementioned lack of variability in the observed optical and near-IR bands of NRAO 150, whereas the radio and mm spectral ranges showed a factor 5 long term variability. This can be easily explained if this observed optical and near-IR (rest frame opticalUV) emission is produced in the accretion disk and not in the relativistic jets where the extreme variability originates.

\subsection{Source classification}

From the VLBI point of view, NRAO 150 shows a one-sided powerful relativistic-superluminal jet typical of blazars, which indicates that the axis must be aligned close to the line of sight. Its radio spectrum is typically flat, which classifies the source as a flat spectrum radio quasar (FSRQ). The optical rest-frame spectrum contains intense and broad $\left(\sim 5000 \mathrm{~km} \mathrm{~s}^{-1}\right) \mathrm{H}_{\alpha}$ and $\mathrm{H}_{\beta}$ emission, so the source is a type $1 \mathrm{AGN}$, consistent with the disk axis being aligned close to the line of sight according to the standard AGN unification scenario (Urry \& Padovani 1995). The optical and radio properties are both consistent given the orientation of the radio jet and the accretion disk. That the forbidden [OIII] lines are weak points to a very luminous central engine, which is also consistent with the high Eddington rate determined from the optical/UV luminosity relative to the $\mathrm{BH}$ mass. The high accretion rate is consistent with the high radio loudness of NRAO 150. The BH mass is also among the highest in value derived for quasars (Vestergaard \& Peterson 2006; Vestergaard et al. 2008; Ghisellini et al. 2009b, and references therein).

\section{Summary and conclusions}

We have determined, for the first time, the cosmological distance of NRAO 150, one of the brightest radio to mm AGN sources in the northern sky, by means of its spectroscopic red$\operatorname{shift}\left(z=1.517\right.$ or $\left.d_{\mathrm{L}}=11.2 \times 10^{4} \mathrm{Mpc}\right)$. Given the low Galactic latitude of the source, its optical spectral lines have remained hidden from us for decades. The new near-IR spectra presented here have detected very intense $\mathrm{H} \alpha$ emission and weaker $\mathrm{H} \beta$ and FeII blended lines.

The measured line width of $\mathrm{H} \alpha$ implies that NRAO 150 is a broad-line AGN. Its disk axis must be oriented close to the line of sight, indicating that it is a powerful one-sided superluminal radio-mm jet. Based also on its radio to $\mathrm{mm}$ spectral and variability properties (including those observed with VLBI), NRAO 150 can be classified as a FSRQ blazar. In agreement with previous observations of high redshift and highly luminous quasars, the optical rest-frame spectrum of the source exhibit weak or absent spectral features typical of the NLR, such as [OIII] $\lambda 5007 \AA$. This implies that the highest luminosity accretion disks (and probably the most massive $\mathrm{BH}$ ) probably experience the unbounding of their NLR by accretion disk radiation.

Using empirical relationships between $\mathrm{H} \beta$ line width and luminosity we estimate that the central engine in NRAO 150 cotains a massive black hole of mass $\sim 5 \times 10^{9} M_{\odot}$. The radio to X-rays SED of the source exhibit two prominent bumps: one peaking at millimetre wavelengths - typical of synchrotron radiation from high power blazar jets - and another in the near-UV ( $2000 \AA$ ) that is attributable to thermal emission from the accretion disk. The good spectral coverage of the disk emission allows us to measure reliably the bolometric luminosity of the disk, which turns out to be accreted at $\sim 30 \%$ of the Eddington rate. This, the high $\mathrm{BH}$ mass of the source, its prominent BLR line luminosity, and its highly luminous synchrotron spectrum implies that NRAO 150 is one of the most powerful FSRQ blazars. These sources are also the most luminous hard $\mathrm{X}$-ray and $\gamma$-ray inverse-Compton blazar emitters (Ghisellini \& Tavecchio 2009; Ghisellini et al. 2009a,b), and are routinely monitored by high energy space observatories such as Fermi. We predict that NRAO 150 is one of these sources. However, it has not yet been detected in $\gamma$-rays. The low Galactic latitude of the source presents a challenge for $\gamma$-rays observatories to detect it. If this were possible in the future, modeling of the whole broad-band spectrum SED would allow us to investigate further the intrinsic physical parameters of this powerful blazar.

Acknowledgements. Financial support by the grant AYA2004-03136 from Plan Nacional de Astronomía y Astrofísica is acknowledged. I.A. acknowledges support by an I3P contract with the Spanish "Consejo Superior de Investigaciones Científicas", and by the Spanish "Ministerio de Ciencia e Innovación" and the European Fund for Regional Development through grant AYA2007-67627C03-03. The Liverpool Telescope is operated on the island of La Palma by Liverpool John Moores University in the Spanish Observatorio del Roque de los Muchachos of the Instituto de Astrofisica de Canarias with financial support from the UK Science and Technology Facilities Council. Some of the data published in this article were acquired with the IAC 80 telescope operated by the Instituto de Astrofísica de Canarias in the Observatorio del Teide. We gratefully acknowledge $\mathrm{H}$. Ungerechts for providing total flux density mm measurements from the general IRAM 30-meter Telescope AGN Monitoring Program. IRAM is supported by INSU/CNRS (France), MPG (Germany) and IGN (Spain).

\section{Appendix A: Galactic extinction correction}

We have already mentioned that the low Galactic latitude of NRAO 150 and its associated high Galactic extinction has defied optical identification and studies of this radio-source. To compute the intrinsic properties of NRAO 150, we have to correct for the Galactic extinction. As a first approach, we have taken the extinction from SIMBAD database, which is estimated from far infrared emission maps build by combining IRAS and COBE/DIRBE data (Schlegel et al. 1998). The quoted value is $E(B-V)=1.474$, which implies that the extinction is between $A_{V}=4.5$ and $A_{K}=0.54$.

To obtain another estimate of the Galactic extinction in the direction of NRAO 150, we compiled a color-magnitude diagram (Fig. A.1) using the near infrared colors ( $J$ and $K$ from 2MASS database Cutri et al. 2003) of neighbouring stars. The color-magnitude $\left(J-K_{\mathrm{s}}, J\right)$ diagram stellar distribution was compared with the theoretical isochrones for 2MASS filters retrieved from Girardi et al. (2002). We attempted to discern part of the Main Sequence in the color-magnitude diagrams and recognized two possible ones (see Fig. A.1), one being, more crowded, and showing an excess $J-K \sim 0.7$ and a second sequence, less populated, with $J-K \sim 1.3$. We identified the first population 


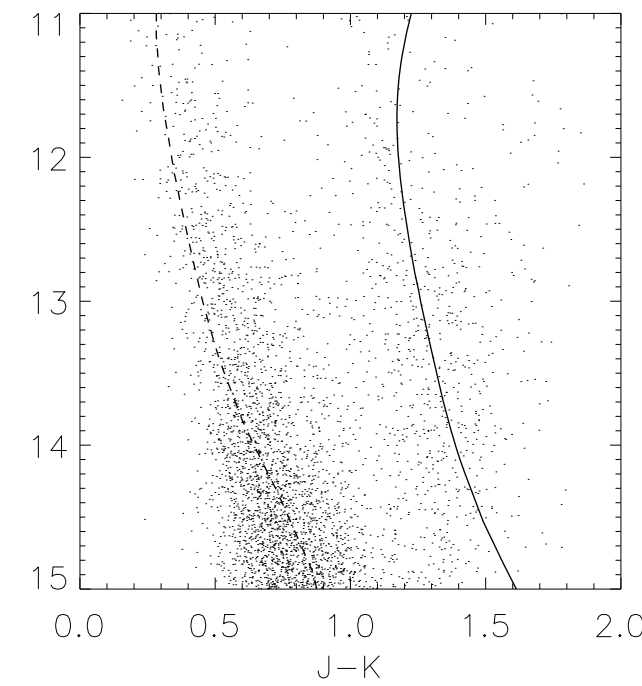

Fig. A.1. Color-magnitude diagram of a field of $30 \times 30$ arcmins, centered on NRAO 150. The two lines correspond to the theoretical Main Sequences of an isochrone with $\log t=9.1 \mathrm{Gyr}$ and $z=0.019$ of Girardi et al. (2002). Continous line fits the more distant and extincted stellar population while dashed line represents the fit to a less extincted population of stars.

to be in front of the Galactic disk and the other to be behind the disk and presenting a higher galactic extinction.

When we assumed the solar metalicity $z=0.019$, we found that the closest possible fits to both stellar populations corresponded to isochrones with $\log t=9.1 \mathrm{Gyr}$ (see Fig. A.1). For these fits, we computed $E(J-K)=1.0 \pm 0.1$ for a stellar population with a distance module of 10.2 , and $E(J-K)=$ $0.12 \pm 0.3$ and a distance module of 9.5 for the less extincted and closer population. We were only interested in the largest value of $E(J-K)$, which implies a value $E(B-V)=1.7 \pm 0.2$ $(E(B-V)=1.69 E(J-K))$. This value is in reasonable agreement with the estimate provided by the SIMBAD database.

\section{References}

Abdo, A. A., Ackermann, M., Ajello, M., et al. 2009, ApJ, 699, 976 Acosta Pulido, J. A., Ballesteros, E., Barreto, M., et al. 2003, INGN, 7, 15A
Agudo, I. 2009, in Approaching Micro-Arcsecond Resolution with VSOP-2: Astrophysics and Technologies, ed. Y. Hagiwara, E. Fomalont, M. Tsuboi, \& Y. Murata, ASP Conf. Ser., 402, 330

Agudo, I., Bach, U., Krichbaum, T. P., et al. 2007, A\&A, 476, L17

Agudo, I., Thum, C., Wiesemeyer, H., \& Krichbaum, T. P. 2010, ApJS, 189, 1 Aller, H. D., Aller, M. F., Latimer, G. E., \& Hodge, P. E. 1985, ApJS, 59, 513

Babbedge, T. S.-R., Rowan-Robinson, M., Gonzalez-Solares, E., et al. 2004, MNRAS, 353, 654

Bennert, N., Falcke, H., Schulz, H., Wilson, A. S., \& Wills, B. J. 2002, ApJ, 574, L105

Bertin, E., \& Arnouts, S. 1996, A\&AS, 117, 393

Boroson, T. A., \& Green, R. F. 1992, ApJS, 80, 109

Cutri, R. M., et al. 2003, VizieR On-line Data Catalog: II/246, Originally published in: University of Massachusetts and Infrared Processing and Analysis Center (IPAC/California Institute of Technology)

D’Ammando, F., Pucella, G., Raiteri, C. M., et al. 2009, A\&A, 508, 181

Fey, A. L., \& Charlot, P. 2000, ApJS, 128, 17

Ghisellini, G., \& Tavecchio, F. 2009, MNRAS, 397, 985

Ghisellini, G., Tavecchio, F., \& Ghirlanda, G. 2009a, MNRAS, 399, 2041

Ghisellini, G., Foschini, L., Volonteri, M., et al. 2009b, MNRAS, 399, L24

Girardi 2002, A\&A, 391, 195

Graham, A. W. 2007, MNRAS, 379, 711

Labita, M., Decarli, R., Treves, A., \& Falomo, R. 2009, MNRAS, 396, 1537

Landau, R., Jones, T. W., Epstein, E. E., et al. 1983, ApJ, 268, 68

Manchado, A., Barreto, M., Acosta-Pulido, J. A., et al. 2004, in Proc. SPIE, 5492, 1094

McLure, R. J., \& Dunlop, J. S. 2004, MNRAS, 352, 1390

Netzer, H., Shemmer, O., Maiolino, R., et al. 2004, ApJ, 614, 558

Oke, J. B., \& Lauer, T. R. 1979, ApJ, 230, 360

Pauliny-Toth, I. I. K., Wade, C. M., \& Heeschen, D. S. 1966, ApJ, 13, 65

Raiteri, C. M., Villata, M., Larionov, V. M., et al. 2007, A\&A, 473, 819

Ramos Almeida, C., Pérez García, A. M., Acosta-Pulido, J. A., et al. 2006, ApJ, 645,148

Ramos Almeida, C., Pérez García, A. M., \& Acosta-Pulido, J. A. 2009, ApJ, 694, 1379

Reuter, H.-P., Kramer, C., Sievers, A., et al. 1997, A\&AS, 122, 271

Schlegel, D. J., Finkbeiner, D. P., \& Davis, M. 1998, ApJ, 500, 525

Shemmer, O., Netzer, H., Maiolino, R., et al. 2004, ApJ, 614, 547

Shen, Y., Greene, J. E., Strauss, M. A., Richards, G. T., \& Schneider, D. P. 2008 , ApJ, 680, 169

Steele, I. A., Smith, R. J., Rees, P. C., et al. 2004, in Ground-based Telescopes, ed. J. M. Oschmann, Jr., Proc. SPIE, 5489, 679

Teräsranta, H., Achren, J., Hanski, M., et al. 2004, A\&A, 427, 769

Teräsranta, H., Wiren, S., Koivisto, P., Saarinen., V., \& Hovatta, T. 2005, A\&A, 440, 409

Vacca, W. D., Cushing, M. C., \& Rayner, J. T. 2003, PASP, 115, 389

Vestergaard, M., \& Peterson, B. M. 2006, ApJ, 641, 689

Vestergaard, M., Fan X., Tremonti, C. A., Osmer, P. S., \& Richards, G. T. 2008 ApJ, 674, L1

Yuan, M. J., \& Wills, B. J. 2003, ApJ, 593, L11

Urry, C. M., \& Padovani, P. 1995, PASP, 107, 803 